\title{
Further Results on Nonlinearly Stretching Permeable Sheets: Analytic Solution for MHD Flow and Mass Transfer
}

\author{
Rafael Cortell \\ Departamento de Física Aplicada, Escuela Técnica Superior de Ingenieros de Caminos, Canales y Puertos, \\ Universidad Politécnica de Valencia, 46022 Valencia, Spain \\ Correspondence should be addressed to Rafael Cortell, rcortell@fis.upv.es
}

Received 24 July 2012; Accepted 28 September 2012

Academic Editor: Ashraf M. Zenkour

Copyright ( $) 2012$ Rafael Cortell. This is an open access article distributed under the Creative Commons Attribution License, which permits unrestricted use, distribution, and reproduction in any medium, provided the original work is properly cited.

\begin{abstract}
The steady magnetohydrodynamic (MHD) flow and mass transfer of an incompressible, viscous, and electrically conducting fluid over a permeable flat surface stretched with nonlinear (quadratic) velocity $u_{w}(x)=a x+c_{0} x^{2}$ and appropriate wall transpiration is investigated. It is shown that the problem permits an analytical solution for the complete set of equations with magnetic field influences when a fictitious presence of a chemical reaction is considered. Velocity and concentration fields are presented through graphs and discussed. The results for both skin friction coefficient $f^{\prime \prime}(0)$ and mass transfer gradient $c^{\prime}(0)$ agree well with numerical results published in the literature.
\end{abstract}

\section{Introduction}

Momentum and mass transport generated by a linearly or nonlinearly stretching surface has extensively been treated over the last few decades due to its relevance to a wide range of industrial applications. In particular, processes involving the mass transfer effect have been recognized as important principally in chemical processing equipment [1]. The heat and mass transfer problem with or without magnetic field, suction or injection, has been analyzed by a great deal of researchers since it has many engineering applications such as production of polymer sheets, crystalline materials and glass sheets, paper and textile industries, and many others. Sakiadis [2] initiated the study of the boundary layer flow over a continuous solid surface moving with constant speed. Crane [3] was the first one who studied the stretching problem taking into account the fluid flow over a linearly stretched surface. The continuous elastic sheet moves in its own plane with a velocity varying linearly with the distance from 
a fixed point. Later, P. S. Gupta and A. S. Gupta [4] extended Crane's work [3] to include suction or blowing, and Vleggaar [5] analyzed the stretching problem with constant surface temperature. After these pioneering works, analyses to the flow and heat/mass transfer over a stretching surface have intensely been made in recent years [1, 6-11]. Moreover, the author of the present study has also analyzed thermal radiation, suction, and viscous dissipation effects on the flow and heat transfer of a power-law fluid over a porous surface [12]. Almost all the aforementioned researches were performed taking into account either a constant value for the velocity wall or a linearly stretching sheet problem (i.e., $u_{w}(x)=a x$ ).

Nevertheless, it needs to be underlined that the quadratic stretching of the sheet may not necessarily be linear. In view of this, a number of studies have appeared in the literature to analyze various different nonlinearly stretching rates; a quadratic stretching function was considered in the early investigation of Takhar et al. [13]. Later, Kumaran and Ramanaiah [14] showed that a quadratic term of stretching could be superposed on the linearly stretching Crane's [3] result by considering a suitable distribution of transpiration along the plate stretched in a nonlinear (quadratic) fashion. The above investigation was recently generalized by Weidman and Magyari [15] to continuous surfaces stretching with arbitrary polynomial velocities, which they designed as superstretching, and then the arbitrary stretching was also extended to micropolar fluids [16]. Cortell [17] proceeded to analyze momentum and heat transport on a continuous planar surface moving in a parallel stream. Mention is also made of the recent published paper by Palani and Kim [18] in which the effects of a first-order homogeneous chemical reaction in an unsteady free convective flow past a vertical plate were numerically investigated. Muhaimin and Kandasamy [19] reported interesting results for mass transfer in free, forced, and mixed convection flows along a porous wedge with magnetic field, chemical reaction, and suction/blowing influences. Further, Abdel-Rahman [20] studied thermal diffusion and MHD for Soret and Dufour's effects on Hiemenz flow and mass transfer with variable viscosity.

Exponentially stretching sheet was studied by Elbashbeshy [21]. Further, the effects of suction/blowing and viscous dissipation on the steady nonlinear viscous flow and heat transfer over a horizontal shrinking permeable sheet were recently studied by Rohni et al. [22].

The present author has also studied $[23,24]$ flow and heat transfer onto a nonlinearly stretching sheet for three different types of thermal boundary conditions on the sheet: constant surface temperature (CST case), prescribed surface temperature (PST case), and prescribed heat flux (PHF case). These investigations were based on the assumption that the effects of thermal radiation and viscous dissipation were enclosed in the energy equation. Flow and heat transfer characteristics in a viscous fluid over a nonlinearly stretching sheet are recently analyzed analytically by Akyildiz and Siginer [25].

Another study which belongs to these classes of problems was recently done by Bataller [26]. He stated a true similarity problem and presented a numerical analysis in connection with the laminar boundary layer flow induced in a quiescent fluid by an impermeable stretching flat surface with velocity $u_{w}(x) \approx x^{1 / 3}$. Realize that, in general, $u_{w}(x)=C x^{k}, k$ being an arbitrary positive constant (not necessarily an integer), as was very recently pointed out by Prasad and Vajravelu [27]. Among others, the quadratic stretching sheet case was devised by Khan and Sanjayanand [28]. The MHD flow of a viscous fluid over a sheet stretching with nonlinear (quadratic) velocities without suction/blowing effects at the surface was recently considered by Raptis and Perdikis [29] and Kelson [30]. These types of fluid flow problems have also been extended by Ahmad and Asghar [31] to a sheet stretching with arbitrary velocities in the case of fluid flow of a second grade fluid. 
Hence, the purpose of the present paper is to extend the most recent studies $[14,29,30]$ to the case of permeable wall with magnetic field influences together with mass transfer. As in Kumaran and Ramanaiah [14] was made, we also adopt both quadratic polynomial velocity and linear mass flux at the surface. However, in the previous study [14], magnetic field influences were not included; hence, the present paper is devoted to analyze MHD viscous incompressible flow and mass transfer with quadratic polynomial velocity at the sheet.

The standard approach here is to find similarity solution for the complete system of governing equations. As a result, in some few cases, the system of partial differential equations permits a similarity transformation to convert it into a system of ordinary differential equations, and then similarity solutions can be found. Although velocity and concentration fields are interdependent (through the relevant parameter $\alpha$ ), we in the following consider the steady MHD viscous flow and mass transfer due to nonlinearly (quadratic) stretching sheet with a fictitious destructive chemical reaction effects when the full set of governing equations (i.e., continuity, momentum, and mass transfers) permits the similarity transformation for various functions of exponential type. On this assumption, a complete set of governing equations can be satisfied, and hence an exact solution for the stated problem can be found. As we will see, different from early works regarding the outlined problem (see, e.g., $[29,30]$ ), a remarkable prescribed wall concentration $c_{w}(x)=$ $A\left(a x+c_{0} x^{2}\right)^{\mathrm{Sc}}$ is assumed which corresponds to the boundary-layer concentration solution $c(x, y)$ at $y=0$, together with an appropriate $x$-dependent wall transpiration $v(x, 0)=v_{w}(x)$. Although these boundary conditions may be difficult to realize in practice, we are carrying out these analyses with a principal emphasis on the similarity solutions for the boundary layer MHD flow and mass transfer, which constitute the basis for real engineering problems. The present author [32] has treated the case of MHD flow and mass transfer for second grade fluid over a linearly stretching sheet with chemically reactive species when the concentration of the reactant is maintained at a prescribed constant wall value $c_{w}$, different from the present boundary assumption in which $c_{w}=c_{w}(x)$.

The problem under consideration is governed by three parameters, that is, the magnetic parameter $N$, the suction/blowing parameter $f_{\mathrm{w}}$, and the Schmidt number Sc. The reaction rate parameter $\beta$ ( $\beta>0$ represents a destructive chemical reaction; $\beta<0$ indicates generative chemical reaction; and $\beta=0$ is the case when a nonreactive species problem takes place) should be obtained as $\beta=N$. Sc. It is hoped that the obtained results will not only provide useful information for applications but also serve as a completion to the related previous studies. On the other hand, in order to assess the accuracy of the presented results, several comparisons are made between the present results and previously published results, for special cases.

\section{The Flow}

Let us suppose steady, constant properties, laminar and two-dimensional flow of an incompressible, electrically conducting, and Boussinesq viscous fluid subject to a transverse uniform magnetic field $B_{0}$ which is applied in the positive $y$-direction past a flat, horizontal, and porous sheet coinciding with the plane $y=0$, the flow being confined to $y>0$. The motion of the fluid is generated due to nonlinearly (quadratic) stretching the sheet with the application of two equal and opposite forces, which are applied along the $x$-axis so that the wall is stretched keeping the origin fixed. The velocity of the fluid far away from the plate is equal to zero, whereas fluid suction/injection is imposed at the plate surface. The magnetic 
Reynolds number is considered to be small so that the induced magnetic field is negligible in comparison to the applied magnetic field. The governing equations are

$$
\begin{gathered}
\frac{\partial u}{\partial x}+\frac{\partial v}{\partial y}=0 \\
u \frac{\partial u}{\partial x}+v \frac{\partial u}{\partial y}=v \frac{\partial^{2} u}{\partial y^{2}}-\frac{\sigma B_{0}^{2}}{\rho} u
\end{gathered}
$$

where $x$ and $y$ are the coordinates along and normal to the sheet, respectively, $u$ and $v$ are the velocity components in $x$ and $y$ directions, $v$ is the kinematic viscosity, $\rho$ is the fluid density, $B_{0}$ is the strength of a uniform magnetic field, which is applied along the $y$-axis, and $\sigma$ is the electric conductivity. The boundary conditions governing the flow subproblem are

$$
\begin{gathered}
u_{w}(x)=a x+c_{0} x^{2}, \quad v=v_{w}(x) \quad \text { at } y=0, \\
u \longrightarrow 0 \quad \text { as } y \longrightarrow \infty .
\end{gathered}
$$

Here $a\left[\mathrm{~s}^{-1}\right], c_{0}\left[\mathrm{~m}^{-1} \mathrm{~s}^{-1}\right]$ are positive nonzero constants, and $u_{w}(x), v_{w}(x)$ are the stretching and transpiration sheet velocities, respectively.

The similarity variable is defined as

$$
\eta=\sqrt{\frac{a}{v}} y
$$

and we also introduce the dimensionless variables $f$ and $g$ related to velocity components as

$$
u=a x f^{\prime}+c_{0} x^{2} g^{\prime}, \quad v=-\sqrt{a v} f-\frac{2 c_{0} x}{\sqrt{a / v}} g
$$

Taking into account (2.4) and (2.5), one further easily can write

$$
\frac{\partial u}{\partial x}=a f^{\prime}+2 c_{0} x g^{\prime} ; \quad \frac{\partial v}{\partial y}=-a f^{\prime}-2 c_{0} x g^{\prime}
$$

and also

$$
\frac{\partial u}{\partial y}=a x f^{\prime \prime} \sqrt{\frac{a}{v}}+c_{0} x^{2} g^{\prime \prime} \sqrt{\frac{a}{v}} ; \quad \frac{\partial^{2} u}{\partial y^{2}}=\frac{a^{2} x}{v} f^{\prime \prime \prime}+\frac{a c_{0} x^{2}}{v} \cdot g^{\prime \prime \prime}
$$

Then, on substitution into (2.2), one can arrive at

$$
a^{2} x\left(f^{\prime \prime \prime}+f f^{\prime \prime}-f^{\prime 2}-N f^{\prime}\right)+c_{0} a x^{2}\left(g^{\prime \prime \prime}+f g^{\prime \prime}-3 f^{\prime} g^{\prime}+2 g f^{\prime \prime}-N g^{\prime}\right)+2 c_{0}^{2} x^{3}\left(g^{\prime 2}-g g^{\prime \prime}\right)=0,
$$


and we directly obtain from (2.8) the three following ordinary differential equations:

$$
\begin{gathered}
f^{\prime \prime \prime}+f f^{\prime \prime}-\left(f^{\prime}\right)^{2}-N f^{\prime}=0, \\
g^{\prime \prime \prime}+f g^{\prime \prime}-3 f^{\prime} g^{\prime}+2 g f^{\prime \prime}-N g^{\prime}=0, \\
g^{\prime 2}-g g^{\prime \prime}=0,
\end{gathered}
$$

where a prime denotes differentiation with respect to the independent similarity variable $\eta$, and $N=\sigma B_{0}^{2} / a \rho$ is the magnetic parameter. It is necessary to note that Kelson [30] revisited [29] in order to include (2.11). On the other hand, realize that the mass conservation equation (2.1) is automatically satisfied (see (2.6)). Equations (2.9)-(2.11) are to be solved subject to the transformed boundary conditions:

$$
\begin{gathered}
f=f_{w}, \quad f^{\prime}=1, \quad g=b, \quad g^{\prime}=1 \quad \text { at } \eta=0, \\
f^{\prime} \longrightarrow 0, \quad g^{\prime} \longrightarrow 0 \quad \text { as } \quad \eta \longrightarrow \infty .
\end{gathered}
$$

It is clear from (2.11) and (2.12) that

$$
g^{\prime \prime}(0)=\frac{1}{b}
$$

An analytical solution of (2.9), (2.10), (2.12), and (2.13) for the cases $N=0, f_{w}=0$, and $b=0$ was recently derived by Kelson [30]; however, the more relevant cases of $N \neq 0$ and $b \neq 0$ have not been treated in [30]. It is clearly necessary to note that Kelson's [30] solution for $g(\eta)$ (i.e., (9) in [30]) does not satisfy (2.11) at $\eta=0$, and then the stated problem would still remain unsolved, and hence all the above justifies our appendix. The novelty here, compared with other works cited above, lies in the attainment of exact solutions describing MHD flow and mass transfer generated by a flat sheet undergoing nonlinear (quadratic) stretching, which we outline in the following.

Fortunately, (2.9) with the boundary conditions $f(0)=f_{w}, f^{\prime}(0)=1$, and $f^{\prime}(\infty)=0$ has an exact solution in the form (see also Cortell [9])

$$
f(\eta)=f_{w}+\frac{1-\exp (-\alpha \eta)}{\alpha}
$$

where

$$
\alpha=\frac{f_{w}+\sqrt{f_{w}^{2}+4(1+N)}}{2} .
$$

Thus, from (2.15) and (2.16), we get a simple exact analytical solution of (2.9) with the above associate boundary conditions and we will utilize this solution for the function $f$. 
On the other hand, (2.11) with the boundary conditions $g(0)=-1 / \alpha, g^{\prime}(0)=1$, $g^{\prime \prime}(0)=-\alpha$, and $g^{\prime}(\infty)=0$ has also an exact solution in the form

$$
g(\eta)=-\frac{\exp (-\alpha \eta)}{\alpha}
$$

where $\alpha$ will be given by (2.16).

Furthermore, on substitution into (2.10) of the functions $f(\eta)$ and $g(\eta)$, which are given by (2.15) and (2.17), respectively, one immediately concludes that (2.10) becomes

$$
\alpha^{2}-f_{w} \alpha-(N+1)=0,
$$

which holds when $\alpha$ is given, precisely, by (2.16). From the above, we can see that (2.9)-(2.11), which govern our stated problem, are satisfied by (2.15), (2.16), and (2.17).

Then, a complete analytical solution for the permeable case when the sheet is stretched in a nonlinear (quadratic) fashion is found as follow:

$$
\begin{gathered}
u(x, y)=\left(a x+c_{0} x^{2}\right) \exp (-\alpha \eta) \\
v(x, y)=-\sqrt{a v}\left[f_{w}+\frac{1-\exp (-\alpha \eta)}{\alpha}\right]+\frac{2 c_{0} x}{\alpha \sqrt{a / v}} \exp (-\alpha \eta)
\end{gathered}
$$

where $\alpha$ is obtained from (2.16).

The associate boundary conditions read

$$
\begin{gathered}
f(0)=f_{w} ; \quad f^{\prime}(0)=1, \quad f^{\prime \prime}(0)=-\alpha \\
f(\infty)=f_{w}+\frac{1}{\alpha} ; \quad f^{\prime}(\infty)=0,
\end{gathered}
$$

for $f(\eta)$,

$$
\begin{gathered}
g(0)=-\frac{1}{\alpha} ; \quad g^{\prime}(0)=1, \quad g^{\prime \prime}(0)=-\alpha \\
g(\infty)=g^{\prime}(\infty)=0,
\end{gathered}
$$

for $g(\eta)$. It is clearly necessary to note that the latter can also be obtained from (2.12)-(2.14) setting $b=-1 / \alpha$.

Based on the second equation of (2.19), the transverse velocity component of the edge of the boundary layer $v_{\infty}$ (i.e., the entrainment velocity) is obtained as

$$
v(x, \infty)=v_{\infty}=-\sqrt{a v}\left[f_{w}+\frac{1}{\alpha}\right] .
$$

This velocity component $v_{\infty}$ depends on $f_{w}, \alpha$, and $N$ flow parameters, and it is related to the amount of fluid which is entrained by the movement of the surface (dragged by the sheet). 


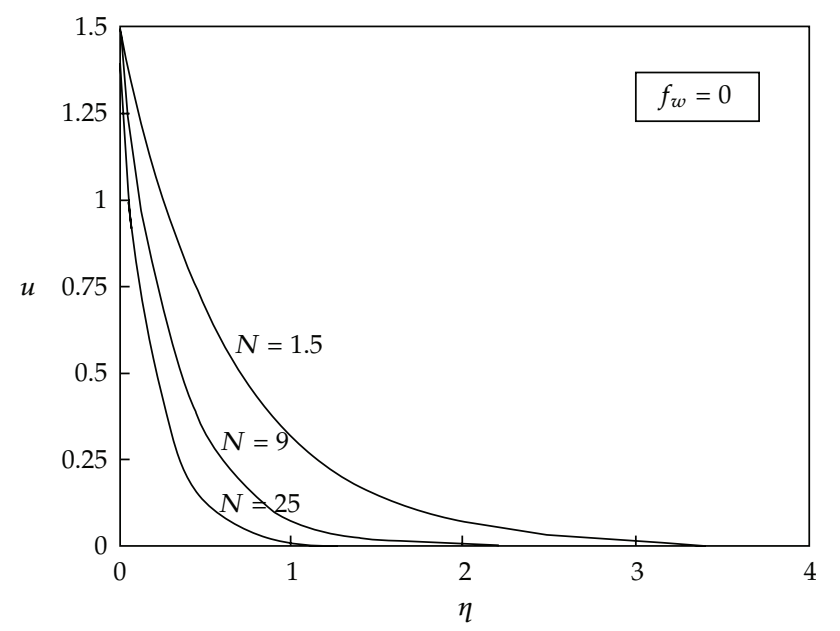

Figure 1: Velocity profiles $u$ for several values of $N$ when $f_{w}=0$.

In addition, one immediately obtains from the second equation of (2.5) and (2.19)

$$
v(x, 0)=v_{w}(x)=-\sqrt{a v} f_{w}-\frac{2 c_{0} x}{\sqrt{a / v}} g(0)=-\sqrt{a v} f_{w}+\frac{2 c_{0} x}{\alpha \sqrt{a / v}} .
$$

One also sees from (2.23) that the suction/injection imposed at the plate surface $v_{w}(x)$ (i.e., the transpiration velocity) turns out in a $x$-dependent blowing when $f_{w}$ is either zero or negative.

Figures 1-3 extract graphical information about the effects of $f_{w}$ and $N$ onto velocity profiles when $a=0.5 \mathrm{~s}^{-1}, c_{0}=1 \mathrm{~m}^{-1} \mathrm{~s}^{-1}$, and $x=1 \mathrm{~m}$. Figure 1 shows the effect of the magnetic parameter $N$ onto velocity profiles when $f_{w}=0$. It can be seen that the velocity $u$ decreases when the magnetic parameter $N$ increases, and then the boundary layer thickness shrinks. In Figure 2, we have plotted the velocity profiles $u$ showing the effect of the parameter $f_{w}$ at nonzero magnetic field, say $N=1.5$. One can see from this graph that as the parameter $f_{w}$ decreases from positive to negative, the magnitude of the velocity largely increases in the boundary flow region.

On the other hand, one can analyse, from Figure 3, the influence of the parameter $N$ and sign of $f_{w}$ on velocity profiles graphically. The velocity profiles boost as the parameter $f_{w}$ decreases from positive to negative, and also the magnetic field effects acquire a major significance when $f_{w}$ is negative (i.e., variable blowing). It is also found that for strong suction $\left(f_{w}>0\right)$ the velocity decreases in the boundary flow region. An explanation for this behavior is that the fluid is brought closer to the sheet in the case of suction, and the opposite behavior is observed, however, in the case of blowing $\left(f_{w}<0\right)$.

It should be noted that (2.8) reduces to the simpler (2.9) for the case when $c_{0}=0$ (i.e., linear stretching). For this special case and in order to asses the accuracy of the present method, we compare values of the skin friction coefficient $f^{\prime \prime}(0)$ between the present results and those of Andersson et al. [33] and Vajravelu et al. [34] for a Newtonian fluid in Table 1. 


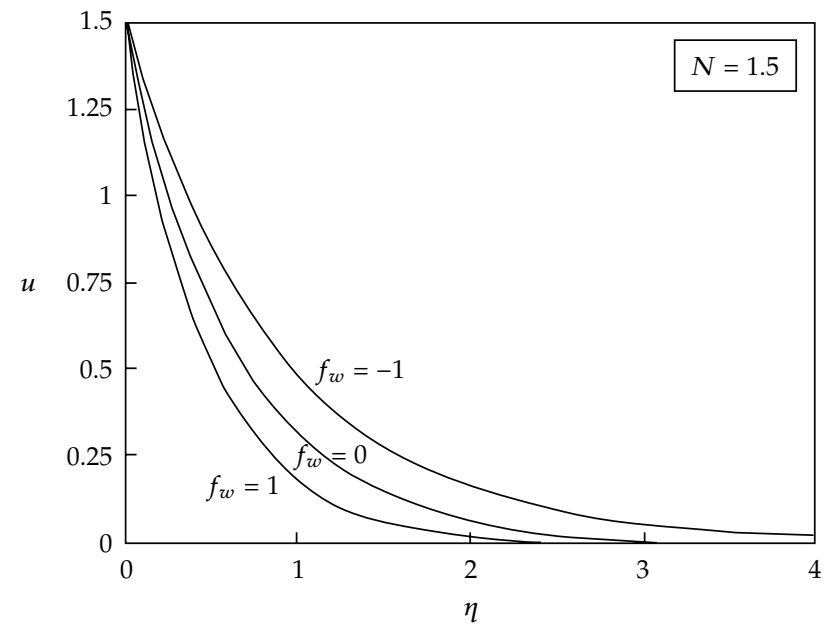

Figure 2: Velocity profiles $u$ for several values of $f_{w}$ when $N=1.5$.

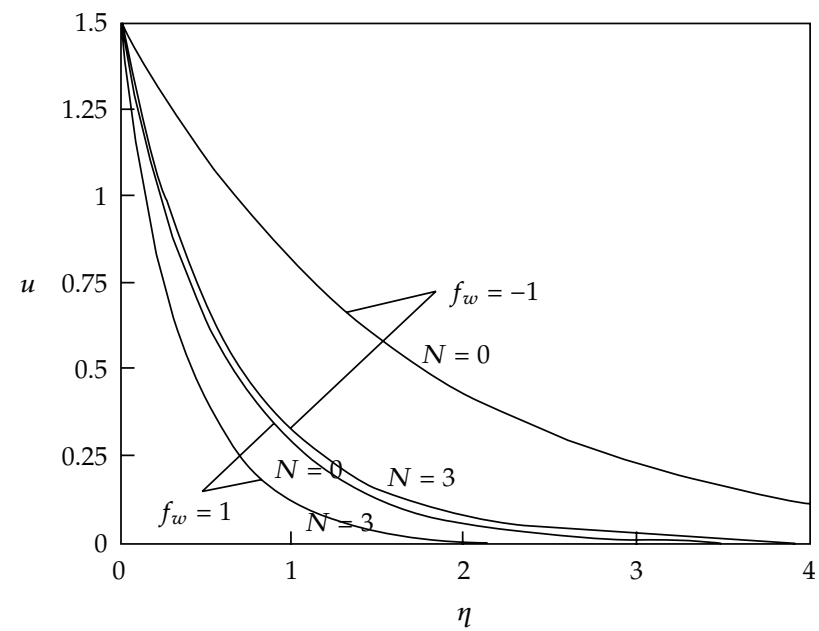

Figure 3: $u$ against $\eta$ for $f_{w}=1,-1$ and $N=0,3$.

\section{The Mass Transfer}

The concentration field $c(x, y)$ is governed by the boundary layer mass diffusion equation (see $[35,36])$ which in the boundary layer approximation reduces to

$$
u \frac{\partial c}{\partial x}+v \frac{\partial c}{\partial y}=D \frac{\partial^{2} c}{\partial y^{2}}-k_{1} c
$$

where $c$ is the concentration of the species of the fluid, $D$ is the diffusion coefficient of the diffusing species in the fluid, and $k_{1}$ denotes the reaction rate constant of the first-order homogeneous and irreversible reaction. The diffusing species can be either destroyed or generated in the homogeneous reaction. The concentration of the reactant will be maintained 
Table 1: Comparison of skin friction coefficient $f^{\prime \prime}(0)$ with that of Vajravelu et al. [34] and Andersson et al. [33] for $c_{0}=0$.

\begin{tabular}{lcc}
\hline & $N$ & $f^{\prime \prime}(0)$ \\
\hline Vajravelu et al. [34] & & -1.0 \\
Andersson et al. [33] & 0.0 & -1.0 \\
Present result & & -1.0 \\
\hline Vajravelu et al. [34] & \multirow{2}{*}{0.5} & -1.2247 \\
Andersson et al. [33] & & -1.2247 \\
Present result & & -1.2247449 \\
\hline
\end{tabular}

at a prescribed wall distribution $c_{w}(x)$ at the surface and is assumed to vanish far away from the surface. As we will outline in the following, the effects onto concentration profiles $c$ of the magnetic parameter $N$ are identical to those in (3.1) due to a first-order destructive chemical reaction, which has been accounted by enclosing the last term in the right-hand side of (3.1). Thus, the appropriate boundary conditions for the concentration (3.1) are given by

$$
\begin{aligned}
& c(x, y)=c_{w}(x) \quad \text { at } y=0, \\
& c(x, y) \longrightarrow 0 \text { as } y \longrightarrow \infty .
\end{aligned}
$$

From (3.1), it is seen that a peculiar analytical solution will be given in the form

$$
c(x, y)=A\left(a x+c_{0} x^{2}\right)^{\mathrm{Sc}} \exp (-\alpha \mathrm{Sc} \eta)
$$

with a wall concentration distribution

$$
c(x, 0)=c_{w}(x)=A\left(a x+c_{0} x^{2}\right)^{\mathrm{Sc}},
$$

where $A$ is a constant and $\mathrm{Sc}=v / D$ is the Schmidt number.

By inserting (2.19) and (3.3) into (3.1), we get

$$
\alpha^{2}-f_{w} \alpha-1-\frac{\beta}{\mathrm{Sc}}=0
$$

where $\beta=k_{1} / a$ is the reaction rate parameter. Besides, replacing $\beta /$ Sc by $N$ into (3.5), we then find that (2.18) and (3.5) are the same. In other words, the effects of a magnetic field onto concentration distributions can now be analyzed assuming $N=\beta / \mathrm{Sc}$, and then there are no magnetic field effects $(N=0)$ when a nonreactive species problem $(\beta=0)$ takes place.

The nondimensional concentration $c(\eta)=c(x, y) / c(x, 0)$ will be

$$
\begin{gathered}
c(\eta)=\exp (-\alpha \mathrm{Sc} \eta), \\
c=1 \quad \text { at } \eta=0 ; \quad c \longrightarrow 0 \quad \text { as } \eta \longrightarrow \infty .
\end{gathered}
$$


Summarily, the solutions of the corresponding momentum transfer $(\vec{v}=u(x, y) \vec{i}+v(x, y) \vec{j})$ and mass transfer $c(x, y)$ subproblems have been given in the forms

$$
\begin{gathered}
u(x, y)=\left(a x+c_{0} x^{2}\right) \exp (-\alpha \eta) \\
v(x, y)=-\sqrt{a v}\left[f_{w}+\frac{1-\exp (-\alpha \eta)}{\alpha}\right]+\frac{2 c_{0} x}{\alpha \sqrt{a / v}} \exp (-\alpha \eta) \\
c(x, y)=A\left(a x+c_{0} x^{2}\right)^{\mathrm{Sc}} \exp (-\alpha \mathrm{Sc} \eta)
\end{gathered}
$$

where $\alpha$ would satisfy the following two equations:

$$
\begin{gathered}
\alpha^{2}-f_{w} \alpha-(N+1)=0, \\
\alpha^{2}-f_{w} \alpha-1-\frac{\beta}{\mathrm{Sc}}=0 .
\end{gathered}
$$

It is consequently found that the velocity and concentration fields are interdependent so that from (3.8) we get

$$
N=\frac{\beta}{S_{c}}, \quad \beta>0
$$

and therefore the parameters $N, \beta$, and Sc are related.

Also, from (3.9), one deduces that the concentration solutions exist only in the case of a fictitious destructive chemical reaction (i.e., reactant consuming $(\beta>0)$, chemical reactions).

On the other hand, the wall transpiration and the prescribed wall concentration will be given in the form

$$
\begin{gathered}
v(x, 0)=v_{w}(x)=-\sqrt{a v} f_{w}+\frac{2 c_{0} x}{\alpha \sqrt{a / v}} \\
c(x, 0)=c_{w}(x)=A\left(a x+c_{0} x^{2}\right)^{\mathrm{Sc}} .
\end{gathered}
$$

Furthermore, we note that from the first equation of (3.7) the dimensionless velocity can be expressed as $h(\eta)=u(x, y) / u_{w}(x)=\exp (-\alpha \eta)$ and from the third equation of (3.7) we have $c(\eta)=c(x, y) / c(x, 0)=\exp (-\alpha \mathrm{Sc} \eta)$. It is then obvious that the velocity profile $h(\eta)$ is identical to the concentration profile $c(\eta)$ for Schmidt number unity (i.e., $h(\eta)=c(\eta)=\exp (-\alpha \eta))$. On the other hand, will result that the skin friction coefficient $h^{\prime}(0)=-\alpha$ appears to be always negative and that means the stretched surface exerts a drag force on the fluid. Physically, this is reasonable since the development of the momentum boundary layer is generated solely by the stretching surface. We also note that the Schmidt number Sc gives no influences on the velocity boundary layer, which is clear from (3.7). 


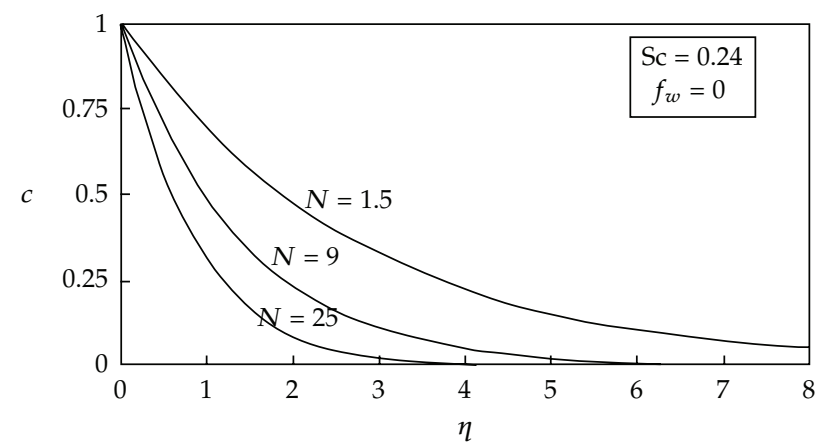

Figure 4: Concentration profiles for quadratic stretching and several values of the magnetic parameter $N$ when $f_{w}=0$ and $\mathrm{Sc}=0.24$.

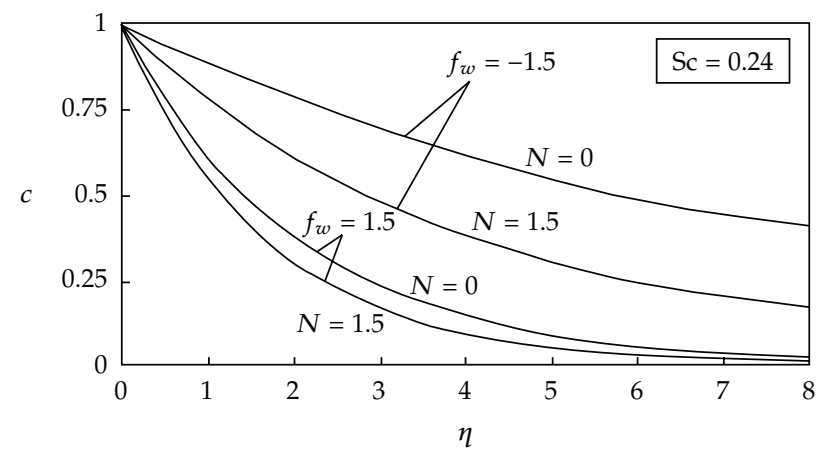

Figure 5: Concentration profiles for quadratic stretching and two values of $f_{w}$ and $N$ when Sc $=0.24$.

Bearing in mind (2.16), (2.19), and (3.9), we get

$$
c(\eta)=\frac{c(x, y)}{c(x, 0)}=\exp (-\alpha \mathrm{Sc} \eta)=\exp \left(-\frac{f_{w}+\sqrt{f_{w}{ }^{2}+4(1+N)}}{2} \mathrm{Sc} \eta\right) .
$$

In order to more fully characterize the behavior of the concentration profiles for a viscous fluid over a nonlinear (quadratic) permeable stretching sheet, with respect to the magnetic parameter $N$, we have depicted in Figure 4 the variation of $c(\eta)$ for three values of $N$ at $\mathrm{Sc}=0.24$ (representing diffusing chemical species of $\mathrm{H}_{2}$ in air) and $f_{w}=0$ (impermeable surface). It can be seen that the concentration $c$ decreases when the magnetic parameter $N$ increases. Further, Figure 5 graphically confirms the later for all $f_{w}$.

Furthermore, as expected, at nonzero magnetic field, say $N=0.5$, Figure 6 shows that an increase in Schmidt number Sc yields a decrease in concentration profiles, whereas for fixed $\mathrm{Sc}$, as the parameter $f_{w}$ decreases from positive to negative, the magnitude of the concentration increases in the boundary flow region. All of these tendencies are consistent with the results of Vajravelu et al. [34].

Finally, we also graphically present the effects of an increase in the magnetic field intensity (through $N$ parameter) onto concentration profiles for both suction and injection situations and for three selected Schmidt number Sc values, say 0.62, 0.78, and 2.62, which 


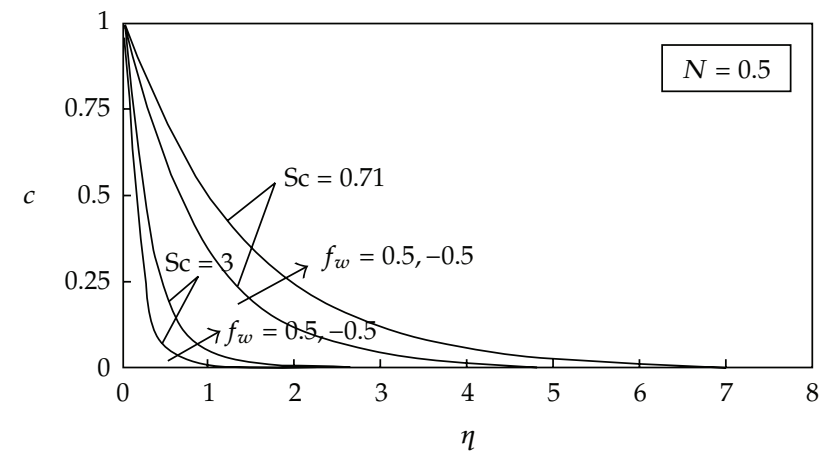

Figure 6: Concentration profiles for quadratic stretching and two values of Sc and $f_{w}$ when $N=0.5$.

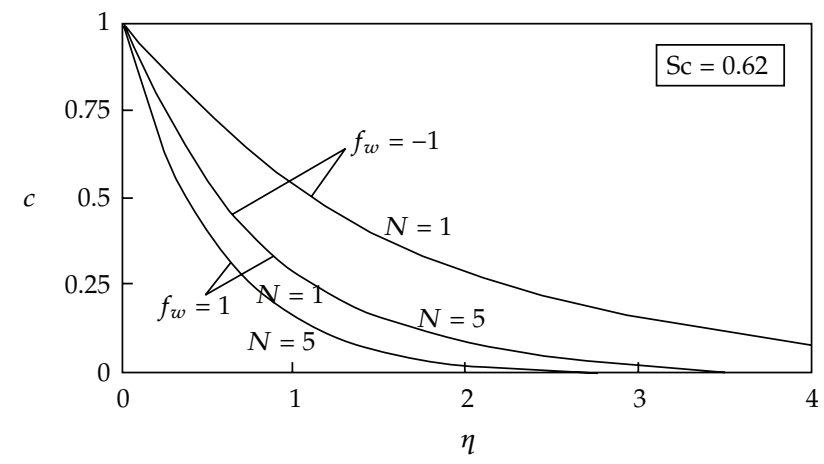

Figure 7: $c$ against $\eta$ for $f_{w}=1,-1$ and $N=1,5$ when $S c=0.62$.

correspond to diffusing chemical species in air of practical interest like $\mathrm{H}_{2} \mathrm{O}, \mathrm{NH}_{3}$, and propyl benzene, respectively [37]. It is found that, irrespective of Sc, an increase in magnetic field intensity causes a decrease in the concentration boundary layer thickness. Moreover, as the Schmidt number Sc increases due to a diminution in the chemical species molecular diffusivity, the thickness of the concentration boundary layer shrinks (see Figures 7, 8 and 9).

In addition, to show the excellent agreement between the present results and those earlier ones from the open literature, use is made of the relevant work by Vajravelu et al. [34], who studied steady MHD non-Newtonian power-law fluid flow over an impermeable linearly stretching sheet with species concentration and chemical reaction. For comparison, one should take the power-law index $n=1$, when the fluid is simply a Newtonian fluid. In [34], the continuous stretching sheet is assumed to have species diffusion $c_{w}(x)=C_{\infty}+$ $E(x / l)^{r}$ where $x$ is the distance from the slit, $l$ is a characteristic length, $E$ is a constant, $r$ is the species concentration parameter, $c_{w}(x)$ is the concentration at the plate, and $C_{\infty}$ indicates the same but far away from the plate surface. Use is made in the present work of $c_{w}(x)=$ $A\left(a x+c_{0} x^{2}\right)^{\mathrm{Sc}}$, cf. (3.4). Hence, on comparison, it should be $r=1 ; G c_{x}=0$ (in [34]) and $\mathrm{Sc}=1 ; \quad c_{0}=0$ (in the present paper). Further, (3.11) with $f_{w}=0$ will provide the present results.

From Table 2, one can see the excellent agreement between Vajravelu et al.'s [34] results, which were obtained numerically by a finite difference scheme known as the KellerBox method and the results of the analytical solution. 


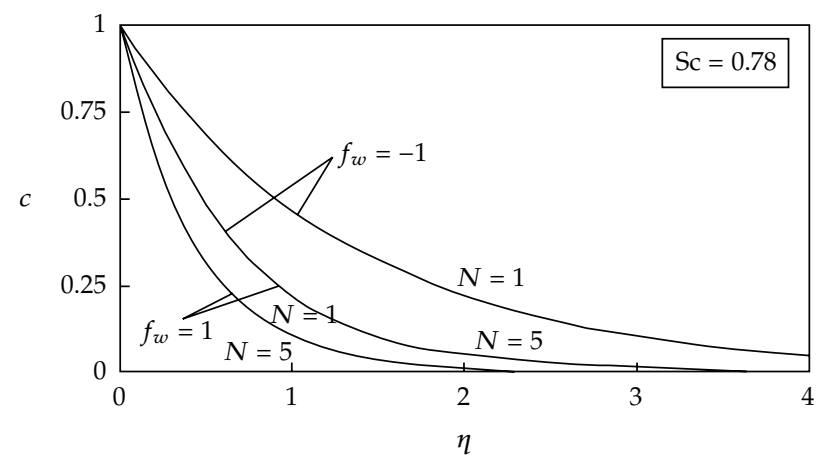

Figure 8: $c$ against $\eta$ for $f_{w}=1,-1$ and $N=1,5$ when $\mathrm{Sc}=0.78$.

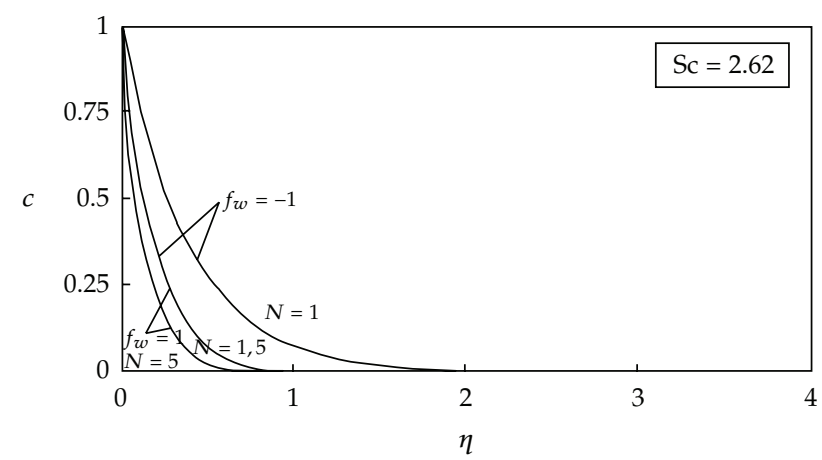

Figure 9: $c$ against $\eta$ for $f_{w}=1,-1$ and $N=1,5$ when $\mathrm{Sc}=2.62$.

\section{Conclusion}

The exact solution corresponding to the motion and mass transfer of a viscous fluid over a permeable nonlinearly (quadratic) stretching sheet is established. The effects of an applied transverse magnetic field are also considered. A suitable $x$-dependent distribution of the transpiration velocity $v_{w}(x)$ should be applied together with a variable prescribed wall concentration $c_{w}(x)$. Both velocity and concentration profiles are interdependent through the relevant parameter $\alpha$. The current analytical study can be considered to be more general than the existing studies due to the fact that magnetic field effects can now be analyzed. The velocity and concentration profiles strongly depend upon the parameters $f_{w}$, Sc, and $N$ and tend to zero at infinity asymptotically. The main results of the paper can be summarized as follows.

(1) As the parameter $f_{w}$ decreases from positive to negative, the magnitude of the velocity largely increases in the boundary flow region.

(2) The velocity and concentration decrease as $N$ increases.

(3) As the parameter $f_{w}$ decreases from positive to negative, the magnitude of the concentration increases in the boundary flow region.

(4) An augment in the Schmidt number Sc shrinks the concentration profiles.

(5) The magnetic field effects on velocity and concentration profiles acquire a major significance when $f_{w}$ is negative (blowing). 
Table 2: Comparison of mass transfer gradient $c^{\prime}(0)$ results with those of Vajravelu et al. [34] for $c_{0}=0$.

\begin{tabular}{lcccc}
\hline & Sc & $\beta$ & $N=(\beta / \mathrm{Sc})$ & $c^{\prime}(0)$ \\
\hline $\begin{array}{l}\text { Vajravelu et al. [34] } \\
\text { Present result }\end{array}$ & 1 & 1 & 1 & -1.414214 \\
Vajravelu et al. [34] & 1 & & -1.4142135 \\
Present result & & 0 & 1.5 & -1.00002313 \\
& 1 & 1.5 & 2 & -1.0 \\
Present results & 1 & 2 & 3 & -1.5811388 \\
& 1 & 3 & 5 & -1.7320508 \\
& 1 & 5 & & -2.0 \\
& & & & -2.4494897 \\
\hline
\end{tabular}

(6) For $\mathrm{Sc}=1$, the dimensionless velocity and concentration profiles are identical.

(7) For the case of Sc $>1$, the dimensionless concentration profile $c(\eta)$ is smaller in comparison to the dimensionless velocity profile $h(\eta)$, and this trend is reversed for the case of $S c<1$.

(8) The Schmidt number Sc exerts no effects on the momentum boundary layer.

\section{Appendix}

Our aim in this appendix is to interpret the obtained results of Kelson [30] and to present a formulation to the stated problem (i.e., similarity solutions for MHD flow due to a permeable and nonlinearly (quadratic) stretching sheet). In [30], an exact solution of (2.9), (2.10), (2.12), and (2.13) for the case $N=0, f_{w}=0$, and $b=0$ is presented as

$$
\begin{gathered}
f(\eta)=1-\exp (-\eta) \\
g(\eta)=\frac{2+(4 \eta-1) \cdot \exp (-\eta)-\exp (-2 \eta)}{7}
\end{gathered}
$$

and one immediately sees that (A.2) does not satisfy (2.11); one can therefore point out that the stated problem would remain unsolved. Different from (A.2), our purpose for the function $\mathrm{g}$ is given by (2.17) with $\alpha=1$,

$$
g(\eta)=-\exp (-\eta)
$$

and then $g(0)=b=-1, g^{\prime}(0)=1$. Further, we have from (2.15)

$$
f(\eta)=1-\exp (-\eta)
$$

which coincides with (8) of [30]. One can then see how (A.3) and (A.4) constitute a solution of (2.9)-(2.13).That is, by replacing (A.2) in [30] with (A.3), one can study the stated problem for the cases $N=0, f_{w}=0$, and $b=-1$. On the other hand, (A.2) and (A.3) are quite different as can be viewed graphically (see Figure 10). 


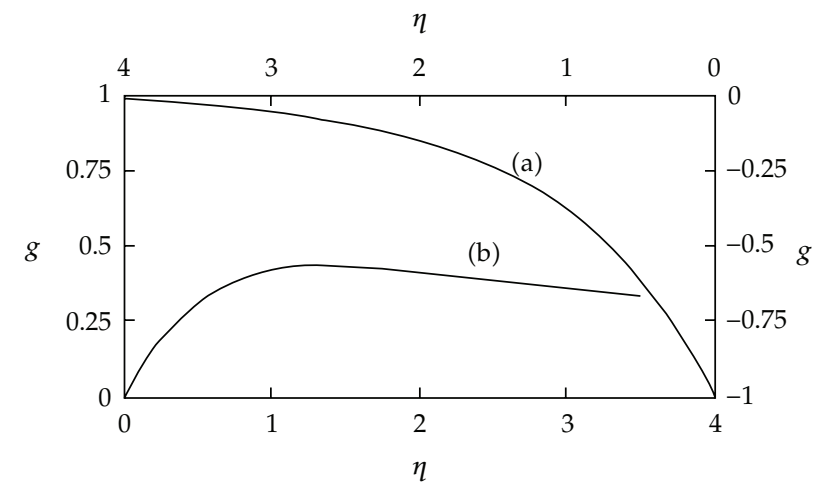

Figure 10: $g$ against $\eta$ : (a) the present solution for function $g$ (A.3); (b) Kelson [30] solution for function $g$ (A.2).

Moreover, in the hypothesis that (A.3) remains unknown for us, one can expand the function $g$ in a Taylor series about $\eta=0$, as it is outlined in the following. It is clear from (2.11) evaluated at $\eta=0$ that

$$
g^{\prime \prime}(0)=\frac{\left(g^{\prime}(0)\right)^{2}}{g(0)}
$$

and incorporating the boundary condition $g^{\prime}(0)=1$, we get

$$
g^{\prime \prime}(0)=\frac{1}{g(0)}
$$

Differentiating (2.11) with respect to $\eta$ results in

$$
g^{\prime} g^{\prime \prime}=g g^{\prime \prime \prime}
$$

This yields

$$
g^{\prime \prime \prime}(0)=\frac{\left(g^{\prime}(0)\right)^{3}}{(g(0))^{2}}=\frac{1}{(g(0))^{2}}
$$

Differentiating (A.7) with respect to $\eta$ will result in

$$
g^{(4)}=\frac{g^{\prime \prime 2}}{g}
$$


Table 3: Comparison of the approximate solution (A.12) with the exact one (A.3) for the function $g$ when $N=0, f_{w}=0$, and $b=-1$.

\begin{tabular}{lcc}
\hline$\eta$ & $(\mathrm{A} .3)$ & $(\mathrm{A} .12)$ \\
\hline 0 & -1 & -1 \\
0.15 & -0.860708 & -0.860708 \\
0.20 & -0.818730 & -0.818736 \\
0.30 & -0.740818 & -0.740837 \\
0.40 & -0.670320 & -0.670397 \\
0.50 & -0.606530 & -0.606770 \\
\hline
\end{tabular}

and hence (A.9) evaluated at $\eta=0$ yields

$$
g^{(4)}(0)=\frac{\left(g^{\prime \prime}(0)\right)^{2}}{g(0)}=\frac{1}{(g(0))^{3}} .
$$

Applying the Taylor series, we get

$$
g(\eta)=g(0)+g^{\prime}(0) \eta+\frac{1}{2} g^{\prime \prime}(0) \eta^{2}+\frac{1}{6} g^{\prime \prime \prime}(0) \eta^{3}+\frac{1}{24} g^{(4)}(0) \eta^{4}+\cdots
$$

Incorporating the boundary condition $g(0)=-1$, all the above yield

$$
g(\eta)=-1+\eta-\frac{1}{2} \eta^{2}+\frac{1}{6} \eta^{3}-\frac{1}{24} \eta^{4}+\cdots
$$

A comparison of approximate solution for sufficiently small values of $\eta,($ A.12) with the exact solution (A.3), is given in Table 3, showing excellent agreement. Obviously, if the solution procedure continues, the accuracy will improve.

Bearing in mind (3.7), the velocity components $u, v$ can be transcribed in the forms

$$
\begin{gathered}
u(x, y)=\left(a x+c_{0} x^{2}\right) \exp (-\eta), \\
v(x, y)=-\sqrt{a v}[1-\exp (-\eta)]+\frac{2 c_{0} x}{\sqrt{a / v}} \exp (-\eta),
\end{gathered}
$$

and also from (2.22) and (2.23), we get

$$
\begin{gathered}
v(x, \infty)=v_{\infty}=-\sqrt{a v} \\
v(x, 0)=v_{w}(x)=\frac{2 c_{0} x}{\sqrt{a / v}} .
\end{gathered}
$$




\section{References}

[1] R. Cortell, "Heat transfer in a fluid through a porous medium over a permeable stretching surface with thermal radiation and variable thermal conductivity," Canadian Journal of Chemical Engineering, vol. 90, no. 5, pp. 1347-1355, 2012.

[2] B. C. Sakiadis, "Boundary-layer behaviour on continuous solid surfaces," AIChE Journal, vol. 7, pp. 26-28, 1961.

[3] L. J. Crane, "Flow past a stretching plate," Zeitschrift für angewandte Mathematik und Physik, vol. 21, no. 4, pp. 645-647, 1970.

[4] P. S. Gupta and A. S. Gupta, "Heat and mass transfer on a stretching sheet with suction or blowing," Canadian Journal of Chemical Engineering, vol. 55, pp. 744-746, 1977.

[5] J. Vleggaar, "Laminar boundary-layer behaviour on continuous, accelerating surfaces," Chemical Engineering Science, vol. 32, no. 12, pp. 1517-1525, 1977.

[6] R. C. Bataller, "Towards a numerical benchmark for MHD flows of upper-convected Maxwell (UCM) fluids over a porous stretching sheet," Fluid Dynamics and Materials Processing, vol. 6, no. 3, pp. 337350, 2010.

[7] T. Hayat, M. Qasim, and Z. Abbas, "Homotopy solution for the unsteady three-dimensional MHD flow and mass transfer in a porous space," Communications in Nonlinear Science and Numerical Simulation, vol. 15, no. 9, pp. 2375-2387, 2010.

[8] R. C. Bataller, "Magnetohydrodynamic flow and heat transfer of an upper-convected Maxwell fluid due to a stretching sheet," Fluid Dynamics E Materials Processing, vol. 7, no. 2, pp. 153-173, 2011.

[9] R. Cortell, "Flow and heat transfer of a fluid through a porous medium over a stretching surface with internal heat generation/absorption and suction/blowing," Fluid Dynamics Research, vol. 37, no. 4, pp. 231-245, 2005.

[10] R. Cortell, "Toward an understanding of the motion and mass transfer with chemically reactive species for two classes of viscoelastic fluid over a porous stretching sheet," Chemical Engineering and Processing, vol. 46, no. 10, pp. 982-989, 2007.

[11] A. Ishak, R. Nazar, N. Bachok, and I. Pop, "Melting heat transfer in steady laminar flow over a moving surface," Heat and Mass Transfer, vol. 46, no. 4, pp. 463-468, 2010.

[12] R. Cortell, "Suction, viscous dissipation and thermal radiation effects on the flow and heat transfer of a power-law fluid past an infinite porous plate," Chemical Engineering Research and Design, vol. 89, no. 1, pp. 85-93, 2011.

[13] H. S. Takhar, A. A. Raptis, and C. P. Perdikis, "MHD asymmetric flow past a semi-infinite moving plate," Acta Mechanica, vol. 65, no. 1-4, pp. 287-290, 1987.

[14] V. Kumaran and G. Ramanaiah, "A note on the flow over a stretching sheet," Acta Mechanica, vol. 116, pp. 229-233, 1996.

[15] P. D. Weidman and E. Magyari, "Generalized Crane flow induced by continuous surfaces stretching with arbitrary velocities," Acta Mechanica, vol. 209, no. 3-4, pp. 353-362, 2010.

[16] E. Magyari and V. Kumaran, "Generalized Crane flows of micropolar fluids," Communications in Nonlinear Science and Numerical Simulation, vol. 15, no. 11, pp. 3237-3240, 2010.

[17] R. Cortell, "Flow and heat transfer in a moving fluid over a moving flat surface," Theoretical and Computational Fluid Dynamics, vol. 21, no. 6, pp. 435-446, 2007.

[18] G. Palani and K. Y. Kim, "On the diffusion of a chemically reactive species in a convective flow past a vertical plate," Journal of Applied Mechanics and Technical Physics, vol. 52, no. 1, pp. 57-66, 2011.

[19] I. Muhaimin and R. Kandasamy, "Local nonsimilarity solution for the impact of a chemical reaction in an MHD mixed convection heat and mass transfer flow over a porous wedge in the presence of suction/injection," Journal of Applied Mechanics and Technical Physics, vol. 51, no. 5, pp. 721-731, 2010.

[20] G. M. Abdel-Rahman, "Thermal-diffusion and MHD for Soret and Dufour's effects on Hiemenz flow and mass transfer of fluid flow through porous medium onto a stretching surface," Physica B, vol. 405, no. 11, pp. 2560-2569, 2010.

[21] E. M. A. Elbashbeshy, "Heat transfer over an exponentially stretching continuous surface with suction," Archives of Mechanics, vol. 53, no. 6, pp. 643-651, 2001.

[22] A. M. Rohni, S. Ahmad, and I. Pop, "Note on Cortell's non-linearly stretching permeable sheet," International Journal of Heat and Mass Transfer, vol. 55, no. 21-22, pp. 5846-5852, 2012.

[23] R. Cortell, "Viscous flow and heat transfer over a nonlinearly stretching sheet," Applied Mathematics and Computation, vol. 184, no. 2, pp. 864-873, 2007.

[24] R. Cortell, "Effects of viscous dissipation and radiation on the thermal boundary layer over a nonlinearly stretching sheet," Physics Letters A, vol. 372, no. 5, pp. 631-636, 2008. 
[25] F. T. Akyildiz and D. A. Siginer, "Galerkin-Legendre spectral method for the velocity and thermal boundary layers over a non-linearly stretching sheet," Nonlinear Analysis. Real World Applications, vol. 11, no. 2, pp. 735-741, 2010.

[26] R. C. Bataller, "Similarity solutions for flow and heat transfer of a quiescent fluid over a nonlinearly stretching surface," Journal of Materials Processing Technology, vol. 203, no. 1-3, pp. 176-183, 2008.

[27] K. V. Prasad and K. Vajravelu, "Heat transfer in the MHD flow of a power law fluid over a nonisothermal stretching sheet," International Journal of Heat and Mass Transfer, vol. 52, no. 21-22, pp. 49564965, 2009.

[28] S. K. Khan and E. Sanjayanand, "Viscoelastic boundary layer MHD flow through a porous medium over a porous quadratic stretching sheet," Archives of Mechanics, vol. 56, no. 3, pp. 191-204, 2004.

[29] A. Raptis and C. Perdikis, "Viscous flow over a non-linearly stretching sheet in the presence of a chemical reaction and magnetic field," International Journal of Non-Linear Mechanics, vol. 41, no. 4, pp. 527-529, 2006.

[30] N. A. Kelson, "Note on similarity solutions for viscous flow over an impermeable and non-linearly (quadratic) stretching sheet," International Journal of Non-Linear Mechanics, vol. 46, no. 8, pp. 10901091, 2011.

[31] A. Ahmad and S. Asghar, "Flow of a second grade fluid over a sheet stretching with arbitrary velocities subject to a transverse magnetic field," Applied Mathematics Letters, vol. 24, no. 11, pp. 19051909, 2011.

[32] R. Cortell, "MHD flow and mass transfer of an electrically conducting fluid of second grade in a porous medium over a stretching sheet with chemically reactive species," Chemical Engineering and Processing, vol. 46, no. 8, pp. 721-728, 2007.

[33] H. I. Andersson, K. H. Bech, and B. S. Dandapat, "Magnetohydrodynamic flow of a power-law fluid over a stretching sheet," International Journal of Non-Linear Mechanics, vol. 27, no. 6, pp. 929-936, 1992.

[34] K. Vajravelu, K. V. Prasad, and N. S. Prasanna Rao, "Diffusion of a chemically reactive species of a power-law fluid past a stretching surface," Computers $\mathcal{E}$ Mathematics with Applications, vol. 62, no. 1, pp. 93-108, 2011.

[35] F. T. Akyildiz, H. Bellout, and K. Vajravelu, "Diffusion of chemically reactive species in a porous medium over a stretching sheet," Journal of Mathematical Analysis and Applications, vol. 320, no. 1, pp. 322-339, 2006.

[36] H. I. Andersson, O. R. Hansen, and B. Holmedal, "Diffusion of a chemically reactive species from a stretching sheet," International Journal of Heat and Mass Transfer, vol. 37, no. 4, pp. 659-664, 1994.

[37] O. D. Makinde, "On MHD heat and mass transfer over a moving vertical plate with a convective surface boundary condition," Canadian Journal of Chemical Engineering, vol. 88, no. 6, pp. 983-990, 2010. 


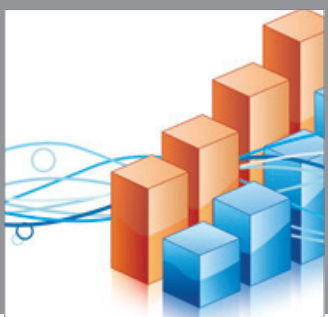

Advances in

Operations Research

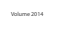

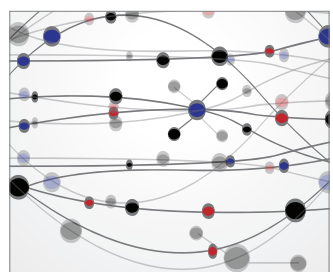

\section{The Scientific} World Journal
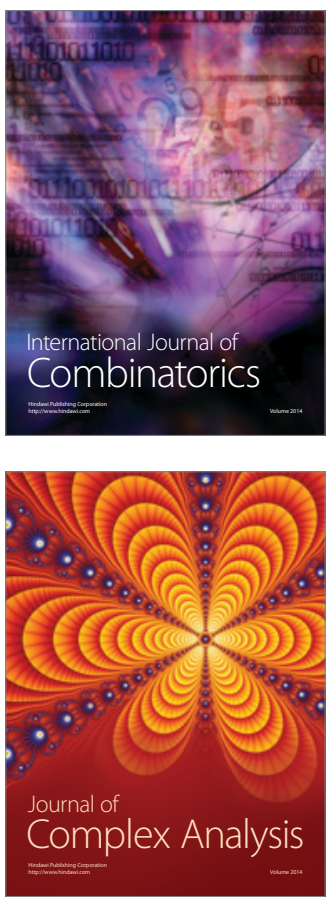

International Journal of

Mathematics and

Mathematical

Sciences
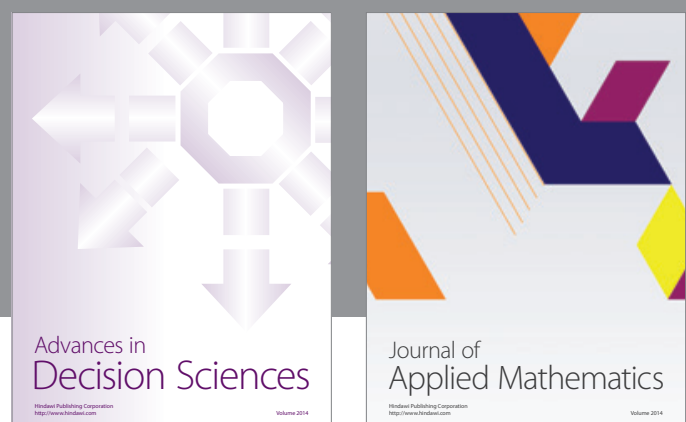

Journal of

Applied Mathematics
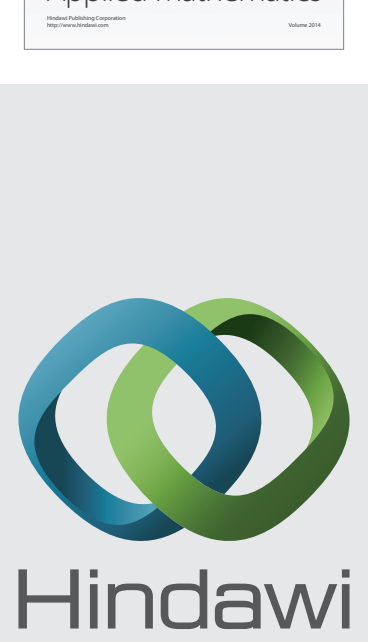

Submit your manuscripts at http://www.hindawi.com
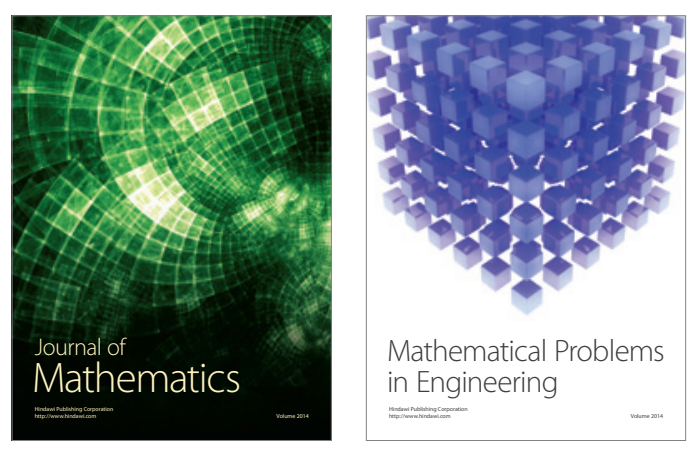

Mathematical Problems in Engineering
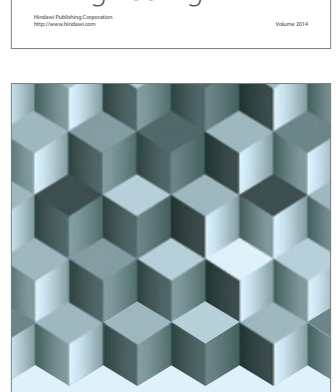

Journal of

Function Spaces
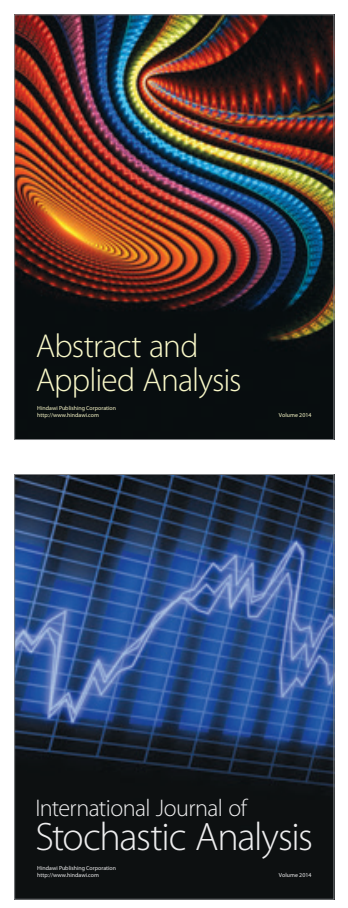



ournal of

Probability and Statistics

Promensencen
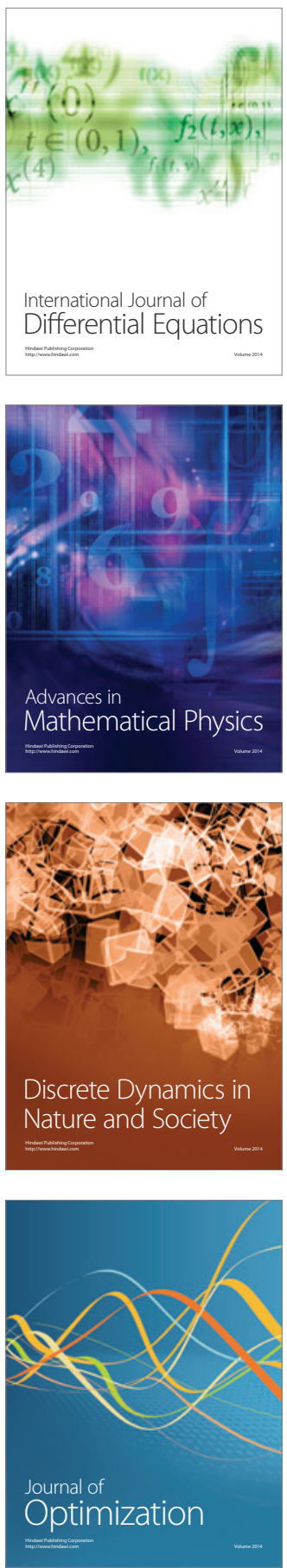